\title{
Interacting Electrons in a Narrow Quantum Hall System
}

\author{
Tapash Chakraborty \\ Max-Planck Institut für Physik Komplexer Systeme \\ Nöthnitzer Strasse 38, D-01187 Dresden, Germany
}

\begin{abstract}
We discuss the electronic properties of a system of interacting electrons in a narrow channel in the quantum Hall effect regime. We demonstrate that the system undergoes phase transitions when the strength of the interaction or the geometry of the channel is varied. The resulting phase diagram contains odd-denominator quantum Hall states and also a stable $v=\frac{1}{2}$ state. The collective mode evaluated at the half-filled case is strikingly similar to that of an odd-denominator state. We discuss the nature of the phases with non-zero total momentum or with no energy gap. We also analyze the spin polarizations of those stable states as a function of temperature. At $v=\frac{1}{2}$ no two-dimensional analog exists, but for the odd-denominator states the temperature dependence closely resemble the ones in the corresponding two dimensional systems where the results have received support from experiments.
\end{abstract}

\section{Introduction}

One of the perhaps most spectacular demonstrations of electron correlations in nature is the fractional quantum Hall effect (FQHE) [1]. It is characterized by Hall plateaus at Landau level filling factors $v=N_{\mathrm{e}} / N_{\mathrm{s}}\left(N_{\mathrm{e}}\right.$ is the number of electrons and $N_{\mathrm{s}}$ is the Landau level degeneracy) corresponding to certain simple fractions with mostly odd denominators. This effect is believed to occur when a two dimensional electron system (2DES) in a strong perpendicular magnetic field and at low temperatures condenses into an incompressible liquid state - the Laughlin state [2]. The FQHE occurs when the chemical potential of the electron system is discontinuous at certain magnetic field dependent densities leading to incompressibility [3]. The energy gap structures in the excitation spectra are also well established for the incompressible states [4]. Interestingly, despite intense activities at the half-filled Landau level [5-7], such a level of understanding has not yet been achieved for the simplest even-denominator state, which is compressible and shows no sign of QHE.

In recent years there has been a great deal of interest in the study of electron correlations in systems where electrons are confined in even lower dimensions [8]. For example, ballistic quantum wires fabricated [9] with long transport mean-free path and very large subband spacings enable one to study effects of electron-electron interactions in onedimensional systems. At high magnetic fields when the radius of the cyclotron orbit - the length scale in the system - is smaller than the width of the channel, it is plausible that the system behaves like a two-dimensional electron gas (2DEG) in the sense that it exhibits QH states. The boundaries of the sample are of course still important in that regime and therefore in theoretical models one should be able to treat the edges and bulk on the same footing. 
Prior to our theoretical work presented here, there were a few earlier attempts to study QHE in one dimension (1D-QHE). Theoretical work on one-dimensional systems with hard wall boundary condition [10] indicated that as the channel width is varied there is a transition between the FQH state and the $2 k_{\mathrm{F}}$-charge density wave state. It was also noticed that the size of the FQHE gap is comparable for the filling factors $v=\frac{1}{2}$ and $v=\frac{1}{3}[10]$, which would mean that just like the stable $\frac{1}{3}$ QHE state (Laughlin state), $v=\frac{1}{2}$ should also exhibit FQHE. Following that theoretical work, observation of incompressible states at specific filling factors in a narrow channel electron system was reported a few years ago [11]. In that experiment, in addition to various odd-denominator fractions, Hall resistance also showed signatures of quantization at the half-filled Landau level. That observation brought into focus the question about the existence and nature of even-denominator states in a narrow channel together with the role of boundaries of the sample. Theoretical interest on 1D-FQHE has been revived by recent work of Yoshioka $[12,13]$ where a parabolic confinement (see below) was used and incompressible states were found at $v=\frac{1}{3}$ and $\frac{2}{3}$, but that approach was somewhat different in spirit than our work described below.

\section{A System of Spinless Electrons}

In our model for the 1D-QHE, electrons (spin polarized) are confined by an electrostatic potential which is parabolic in the lateral direction and flat along the channel, and are also subjected to a strong perpendicular magnetic field. The electrons are considered to be in a cell of length $a$. The width of the cell is determined by the lateral electron density profile which is finite due to the presence of the confinement potential. We impose a periodicity condition along the length of the cell. The total Hamiltonian of the system is

$$
\mathcal{H}=\mathcal{H}_{0}+\mathcal{H}_{\text {int }}
$$

where $\mathcal{H}_{0}$ includes the kinetic energy of $N_{e}$ electrons of effective mass $m^{*}$ and the electrostatic confining potential

$$
\mathcal{H}_{0}=\sum_{i=1}^{N_{\mathrm{e}}}\left[\frac{1}{2 m^{*}}\left(\vec{p}_{i}-e \vec{A}_{i}\right)^{2}+\frac{1}{2} m^{*} \omega_{0}^{2} y_{i}^{2}\right]
$$

with $\vec{A}$ being the vector potential in Landau gauge. The interaction term of the Hamiltonian consists of Coulomb interactions and also terms corresponding to the neutralizing background

$$
\begin{aligned}
\mathcal{H}_{\mathrm{ee}} & =\frac{1}{2} \sum_{i \neq j} \frac{e^{2}}{\varepsilon\left|\vec{r}_{i}-\vec{r}_{j}\right|} \\
\mathcal{H}_{\mathrm{bb}} & =\frac{1}{2} \int d \vec{R} d \vec{R}^{\prime} \frac{e^{2} \rho(\vec{R}) \rho\left(\vec{R}^{\prime}\right)}{\varepsilon\left|\vec{R}-\vec{R}^{\prime}\right|} \\
\mathcal{H}_{\mathrm{eb}} & =-\sum_{i} \int d \vec{R} \frac{e^{2} \rho(\vec{R})}{\varepsilon\left|\vec{R}-\vec{r}_{i}\right|}
\end{aligned}
$$


where $\varepsilon$ is the background dielectric constant and $\rho(\vec{R})$ is the background charge density. The single-electron wave functions labeled by $\kappa=\{m, n\}$ are

$$
\psi_{\mathrm{x}}=\left(\frac{1}{a \sqrt{\pi} \lambda}\right)^{\frac{1}{2}} \exp \left(i k x-\frac{\hat{y}^{2}}{2 \lambda^{2}}\right) H_{n}\left(\frac{\hat{y}}{\lambda}\right)
$$

where $k=(2 \pi / a) m$ is the quantized wave vector and $m$ is the momentum quantum number. The effective Larmor radius for the cyclotron motion is $\lambda=\left(\hbar / m^{*} \Omega\right)^{\frac{1}{2}}$, where $\Omega=\sqrt{\omega_{0}^{2}+\omega_{c}^{2}}$ and $\omega_{c}=e B / m^{*}$ is the cyclotron frequency. The single-electron states Eq. (2.4) are localized around $\hat{y} \equiv y+2 \pi \lambda^{2} m / \gamma a=0$. Here $\gamma=\sqrt{1+\left(\omega_{0} / \omega_{c}\right)^{2}}$ is a dimensionless quantity. The single-electron states for $m$ and $m^{\prime}$ are therefore separated spatially in lateral direction by an amount $\Delta y_{m m^{\prime}}=2 \pi\left(m-m^{\prime}\right) \lambda^{2} / \gamma a$. For a very strong confinement energy with respect to cyclotron energy, spatial separation of the single-electron states vanishes - the system becomes truly one dimensional. On the other hand, when the confinement becomes weaker with respect to cyclotron energy, the states begin to separate spatially and the system becomes quasi-one-dimensional. In (2.4), $H_{n}$ is the Hermite polynomial of order $n$. We consider the limit of a strong magnetic field and therefore, in what follows we set $n=0$ (lowest Landau level). Ignoring the constant Landau level energy the single-electron energies are given by

$$
\mathcal{E}_{m}=\frac{\hbar^{2} k^{2}}{2 m^{*}} \frac{\omega_{0}^{2}}{\Omega^{2}}
$$

It should be pointed out that a related model where the electrons are confined in both $x$ and $y$-directions by a parabolic confinement potential, one gets a system of quantum dots or more popularly termed as artificial atoms [14], whose electronic properties are no less intriguing.

In the non-interacting ground state, $N_{\mathrm{e}}$ electrons occupy the lowest available singleparticle states. It is then reasonable to require that the electron density is symmetric around the $y=0$ axis, i.e., $M=\sum_{j} m_{j}=0$. This symmetry condition holds for odd number of electrons if $m$ is an integer. In that case the boundary condition is periodic: $\psi_{\kappa}(\vec{r}+a \hat{\vec{x}})=\psi_{\kappa}(\vec{r})$. For even number of electrons $m$ has to be a half-odd integer. The boundary condition is then anti-periodic: $\psi_{\mathrm{K}}(\vec{r}+a \hat{\vec{x}})=-\psi_{\mathrm{K}}(\vec{r})$. As we turn on the interelectron interaction electrons start to avoid each other. Increasing the interaction strength with respect to the kinetic energy it becomes energetically favorable for the electrons to occupy also the higher energy single-particle levels in order to reduce the Coulomb repulsion.

Given the single-particle states the Hamiltonian of the many-electron system, projected to the lowest Landau level, is now written

$$
\mathcal{H}=\sum_{m} \mathcal{E}_{m} a_{m}^{\dagger} a_{m}+\sum_{m_{1}, \cdots, m_{4}} \mathcal{A}_{m_{1}, m_{2}, m_{3}, m_{4}} a_{m_{1}}^{\dagger} a_{m_{2}}^{\dagger} a_{m_{3}} a_{m_{4}}
$$

where $a_{m}^{\dagger}\left(a_{m}\right)$ is the creation (annihilation) operator for the state $m$. For the Coulomb potential

$$
v(\vec{r})=\frac{1}{(2 \pi)^{2}} \int d \vec{q} \frac{2 \pi e^{2}}{\varepsilon q} e^{-i \vec{q} \cdot \vec{r}}
$$


where $\vec{q}=\left(q_{x}, q_{y}\right)$, the interaction matrix element is given by

$$
\begin{aligned}
\mathcal{A}_{m_{1}, m_{2}, m_{3}, m_{4}}= & \frac{1}{2} \int d \vec{r}_{1} d \vec{r}_{2} \psi_{m_{1}}^{*}\left(\vec{r}_{1}\right) \psi_{m_{2}}^{*}\left(\vec{r}_{2}\right) v(\vec{r}) \psi_{m_{3}}\left(\vec{r}_{2}\right) \psi_{m_{4}}\left(\vec{r}_{1}\right) \\
= & \frac{1}{2} \frac{e^{2}}{\varepsilon \lambda} \exp \left[-\frac{1}{2}\left(\frac{2 \pi}{\gamma a}\right)^{2}\left(m_{1}-m_{4}\right)^{2}\right] \\
& \times \int d q_{y}^{\prime} \frac{\exp \left[i 2 \pi\left(m_{3}-m_{1}\right) q_{y}^{\prime}\right] \exp \left[-\frac{1}{2}\left(\gamma a q_{y}^{\prime}\right)^{2}\right]}{\sqrt{\left[\frac{2 \pi\left(m_{1}-m_{4}\right)}{\gamma a}\right]^{2}+\left(a q_{y}^{\prime}\right)^{2}}} \\
& \times \delta_{m_{1}+m_{2}, m_{3}+m_{4}}
\end{aligned}
$$

with $q_{y}^{\prime}=q_{y} \lambda^{2} /(\gamma a)$ as the dimensionless integration variable, $\lambda$ is the unit of length and $\mathcal{E}_{\mathrm{c}}=e^{2} / \varepsilon \lambda$ is a measure of the interaction strength. It is to be noted that the diagonal term $m_{1}=m_{4}\left(m_{2}=m_{3}\right)$ diverges as expected for the long-range Coulomb potential. While Yoshioka used a truncated Coulomb potential (section 4), we neutralize the system by embedding the quantum wire into a positively charged background with a density

$$
\rho(\vec{R})=\frac{N_{\mathrm{e}}}{a \sqrt{\pi} d^{\prime}} \exp \left[-\frac{Y^{2}}{d^{\prime 2}}\right] .
$$

The width of the background density profile is denoted by $d^{\prime}$ and in actual calculations we set it equal to the length of the cell. The electrostatic energy of the background charge is

$$
\begin{aligned}
\mathcal{H}_{b} & =\frac{1}{2} \int d \vec{R} d \vec{R}^{\prime} \frac{e^{2} \rho(\vec{R}) \rho\left(\vec{R}^{\prime}\right)}{\varepsilon\left|\vec{R}-\vec{R}^{\prime}\right|} \\
& =\frac{1}{2} N_{\mathrm{e}}^{2} \frac{e^{2}}{\varepsilon \lambda} \frac{1}{a} \int d q_{y}^{\prime} \frac{1}{\left|q_{y}^{\prime}\right|} \exp \left[-\frac{1}{2}\left(\gamma a d^{\prime} q_{y}^{\prime}\right)^{2}\right] .
\end{aligned}
$$

The interaction of electrons with the positive background charge is taken into account by the potential $\mathcal{H}_{\mathrm{eb}}$. In general, the electron-background interaction is a one-body operator with the diagonal matrix element

$$
\begin{aligned}
\mathcal{D}_{m_{1}, m_{2}} & =\int d \vec{r} \psi_{m_{1}}^{*}(\vec{r}) \nu_{e b}(\vec{r}) \psi_{m_{2}}(\vec{r}) \\
& =-\frac{e^{2}}{\varepsilon \lambda} \frac{N_{\mathrm{e}}}{a} \int d q_{y}^{\prime} \frac{1}{\left|q_{y}^{\prime}\right|} \exp \left[i 2 \pi m_{1} q_{y}^{\prime}-\frac{1}{4}\left(\gamma a q_{y}^{\prime}\right)^{2}\left(1+d^{\prime 2}\right)\right] \\
& \times \delta_{m_{1}, m_{2}} .
\end{aligned}
$$

Using the above relations, the final form of the diagonal two-body term is

$$
\begin{aligned}
\mathcal{A}_{m, m^{\prime}, m^{\prime}, m}= & \frac{1}{2} \frac{e^{2}}{\varepsilon \lambda} \frac{1}{a} \int d q_{y}^{\prime} \frac{1}{\left|q_{y}^{\prime}\right|}\left\{\exp \left[i 2 \pi\left(m-m^{\prime}\right) q_{y}^{\prime}-\frac{1}{2}\left(\gamma a q_{y}^{\prime}\right)^{2}\right]\right. \\
& \left.-2 \exp \left[i 2 \pi m q_{y}^{\prime}-\frac{1}{4}\left(1+d^{\prime 2}\right)\left(\gamma a q_{y}^{\prime}\right)^{2}\right]+\exp \left[-\frac{1}{2}\left(\gamma a d^{\prime} q_{y}^{\prime}\right)^{2}\right]\right\}
\end{aligned}
$$




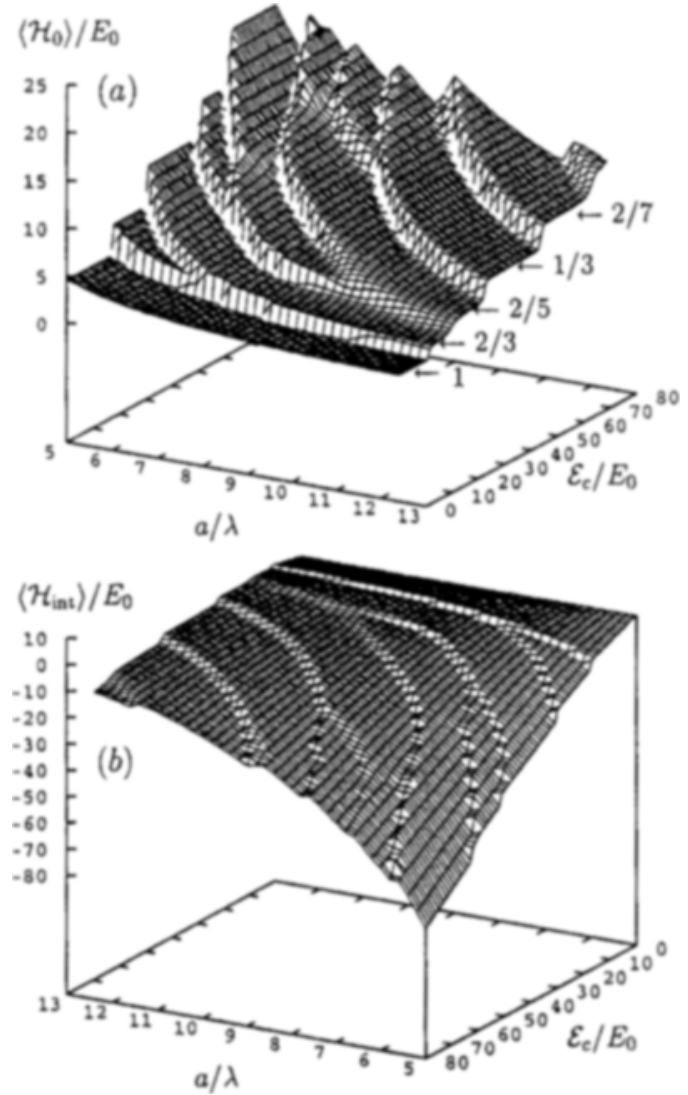

Figure 1

Expectation value of (a) kinetic energy per particle and (b) interaction energy per particle, as a function of $\mathcal{E}_{\mathrm{c}} / E_{0}$ and length of the cell for the state $M=0$. The effective filling factors $v=\frac{1}{3}, \frac{2}{3}, \frac{2}{5}, \frac{2}{7}$ and $v=1$ are also indicated.

where the divergent part of the electron-electron term is now cancelled by the positive background charge. Unlike the other theoretical models of ID-FQHE, in our approach we can therefore include the long-range part of the potential.

\subsection{Filling Factors in a Narrow Channel}

We should point out that evaluation of the filling factor $v$ in a narrow channel is somewhat tricky. Here the single-particle states corresponding to a particular Landau "level" are not degenerate. One possible way would be to calculate the areal electron density and number of fluxes through a unit area and determine $v$ as the ratio of these two quantities. Alternatively, we count the number of occupied states and divide the number of electrons by that. Both methods are somewhat arbitrary: one has to choose properly either the width of the density profile in the first case (we have used below full width at half maximum) or, in the second approach, which state should be considered as occupied. We have checked that both methods agree reasonably well. The $\frac{1}{3} \mathrm{FQH}$ state in the present system is also identified from the momentum distribution function $\langle n(k)\rangle=\left\langle 0\left|a_{k}^{\dagger} a_{k}\right| 0\right\rangle$ by comparing it with that for a Laughlin-like wave function. 


\subsection{Phase Transitions}

In all the numerical results that follow we have used $\omega_{0} / \omega_{c}=0.01$. One has also to be careful about the range of $a / \lambda$, since if the ratio is too large or too small the length or width of the channel gets too large. In those situations, the system becomes truly one dimensional and the electrons prefer to line up along the bottom of the external potential or in the lateral direction respectively. For the various filling factors considered here, the range of $a / \lambda$ is such that the aspect ratio remains usually around unity.

Let us first examine how the translationally invariant state $(M=0)$ changes when we change the strength of interactions with respect to kinetic and potential energies of the electrons, i.e., $\mathcal{E}_{\mathrm{c}} / E_{0}$ (where $E_{0}=\left(\hbar^{2} / 2 m^{*} \lambda^{2}\right)\left(\omega_{0}^{2} / \Omega^{2}\right)$ is the energy unit) and the length of the cell $a$. As we vary $\mathcal{E}_{c} / E_{0}$ while keeping $a$ fixed, the expectation values of the kinetic and potential energies change very abruptly from one value to another. When the calculation is repeated for other fixed values of $a$ we obtain Fig. $1 \mathrm{a}$ and Fig. $1 \mathrm{~b}$ for $\left\langle\mathcal{H}_{0}\right\rangle$ and $\left\langle\mathcal{H}_{\text {int }}\right\rangle$ respectively. These expectation values show rich structures in the parameter space spanned by $a=5, \cdots, 12.4$ and $\mathcal{E}_{c} / E_{0}=0, \cdots, 80[15]$. The two energies $\left\langle\mathcal{H}_{0}\right\rangle$ and $\left\langle\mathcal{H}_{\text {int }}\right\rangle$ jump in opposite directions and therefore the net change in total energy does not clearly show the sudden changes in the $M=0$ state. However, for a much longer system (at a fixed linear density) we expect sharper transitions between the different phases.

As the jump occurs in the parameter space spanned by $\mathcal{E}_{\mathrm{c}} / E_{0}$ and $a$, it indicates a change in the $M=0$ state. Yoshioka identified the filling factors $v=\frac{2}{3}, v=\frac{1}{3}$ and $v=\frac{1}{5}$ FQHE states in a system of six electrons interacting via a truncated Coulomb potential and calculating the overlap with the Laughlin-like wave functions [12]. These states are also realized in our system with real long-range Coulomb potential. The state at $v=\frac{1}{3}$ can also be characterized by calculating the overlap between the Coulomb- $\frac{1}{3}$ state and Haldane's pseudopotential- $\frac{1}{3}$ state [13] (see below). We have checked this overlap in our present system and found it to vary between the values 0.83 and 0.89 at $a=9.5$.

Motivated by indications of the existence of QHE at $v=\frac{1}{2}$ in a narrow channel as mentioned in the introduction, we are interested in going beyond the $v=\frac{1}{3}$ state which seems to be indifferent to the presence of a lateral confinement. In fact, in our quest for a QHE at the half-filled Landau level we are particularly interested to know what happens in between the well established FQHE states. For example, what are the states realized in between the FQHE states $v=\frac{2}{3}$ and $v=\frac{1}{3}$ ? In this region there are clear jumps in both $\left\langle\mathcal{H}_{0}\right\rangle$ and $\left\langle\mathcal{H}_{\text {int }}\right\rangle$. To get further insight on the $M=0$ states realized in the wire, we have investigated the problem of how the electron density profile of the translationally invariant state $(M=0)$ is modified when we change $\mathcal{E}_{\mathrm{c}} / E_{0}$ for a fixed value of $a$.

\subsection{Electron Density}

We have calculated electron density at $\vec{r}$ numerically from [15]

$$
\rho(\vec{r})=\sum_{i, j=1}^{\infty} \Psi_{i}^{*}(\vec{r}) \psi_{j}(\vec{r}) a_{i}^{\dagger} a_{j}
$$



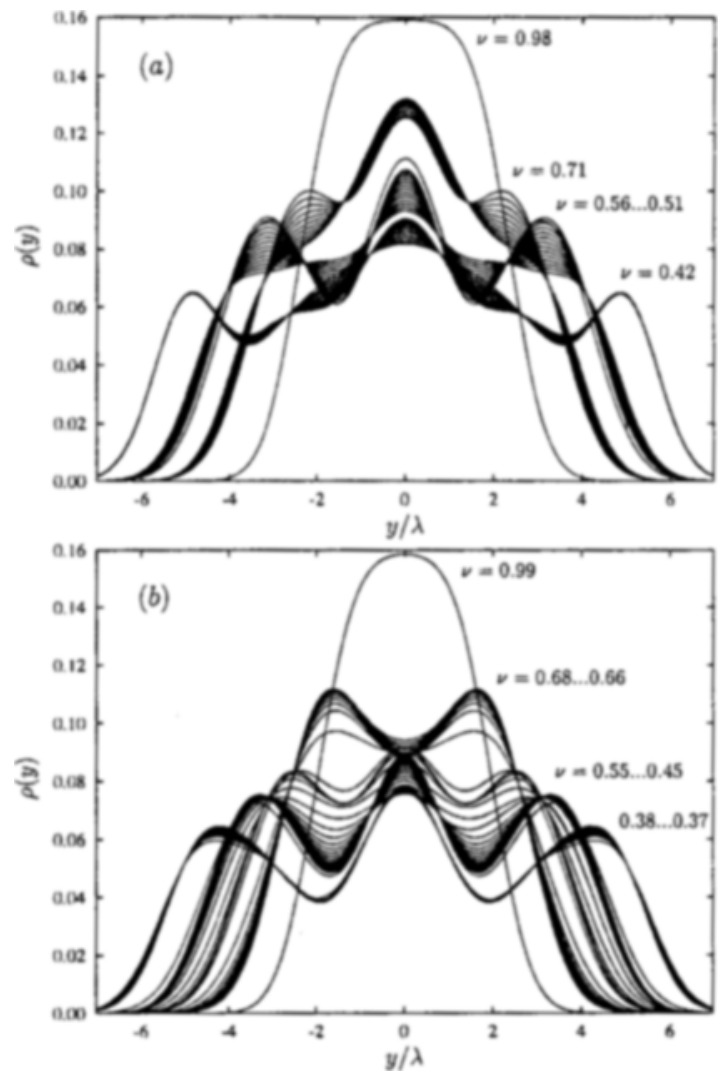

Figure 2

(a) Calculated electon densities in the lateral direction for $a=8$ and the $M=0$ states. (b) The results at $a=9.5$. The effective filling factors are also indicated.

In the $x$-direction the charge density is constant while in the lateral $y$-direction it is modified because of the finite width of the system. Interestingly, as $\mathcal{E}_{c} / E_{0}$ increases the width of the charge density profile also changes abruptly from one value to another (Fig. 2).

The effective filling factors, calculated via $v=2 \pi \lambda^{2} n$ (where $n$ is the number of electrons per unit area and the width of the wire is the full width at half maximum) are found to be $0.98,0.71, \ldots, 0.51$ and 0.42 which are very close to $v=1, \frac{2}{3}, \frac{1}{2}$ and $v=\frac{2}{5}$ in Fig. 1(a) $(a=8)$ and 0.99 and $0.68, \ldots, 0.66$ in Fig. 1(b) $(a=9.5)$ which suggests that these states are $v=1$ and $v=\frac{2}{3}$, respectively. The state which has the effective filling factor $0.38, \ldots, 0.37$ is identified as a $v=\frac{1}{3}$ state also by calculating the Coulomb- $\frac{1}{3}$ state and Haldane's pseudopotential state [13], as discussed above.

\subsection{Phase Diagram}

A phase diagram for the $1 \mathrm{D}-\mathrm{QHE}$ states is then obtained (Fig. 3) by systematically seeking those points in the parameter space spanned by $a$ and $\mathcal{E}_{\mathrm{c}} / E_{0}$ where the ground state has zero total momentum. We then plot the energy gap between this ground state and the first excited state. In Fig. 3 the area of a filled dot is directly proportional to that gap. Clearly, the phase diagram consists of separate regions of several QHE states. Interestingly, there 


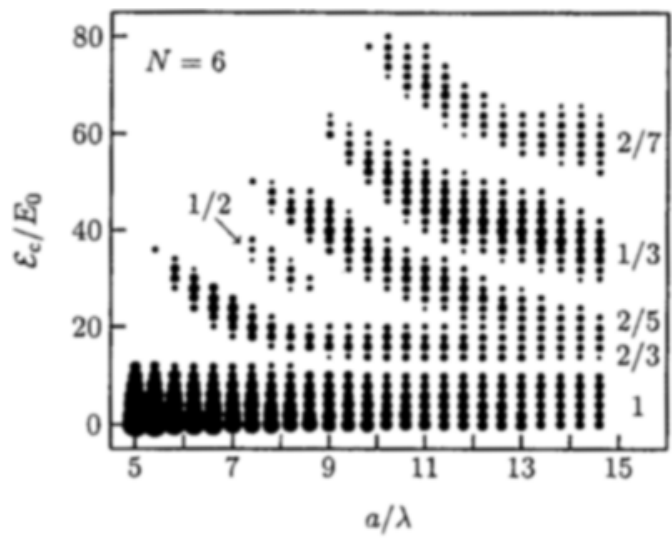

Figure 3

Phase diagram for the FQHE states derived at the effective filling factors $\mathrm{v}=$ $1, \frac{1}{3}, \frac{2}{3}, \frac{2}{3}, \frac{1}{2}, \frac{2}{9}$.

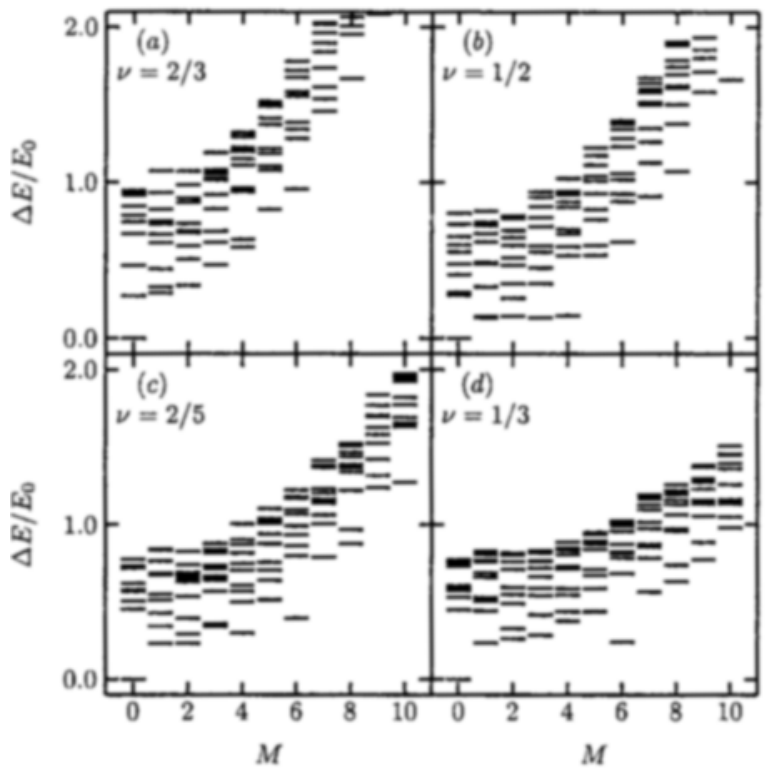

Figure 4

Energy spectra calculated at $\left(a, \mathcal{E}_{c} / E_{0}\right)$ and $\mathrm{v}$ : (a) $(6.8,24), v=\frac{2}{3}$, (b) $(7.6,36), \mathrm{v}=\frac{1}{2},(\mathrm{c})$ $(9.2,40), v=\frac{2}{3}$ and (d) $(12.2,42), v=\frac{1}{3}$.

is also a distinct region for the even-denominator state $v=\frac{1}{2}$ [15]. The phase diagram is dominated by two QHE states, viz., $v=1$ and $v=\frac{1}{3}$ which are also the predominant $Q H$ states observed experimentally in a $2 \mathrm{DEG}$.

\subsection{Collective Modes}

Figure 4 depicts the energy spectra for the states $v=\frac{2}{3}, \frac{1}{2}, \frac{2}{5}$ and $v=\frac{1}{3}$. These states are chosen from the phase diagram (Fig. 3) at the points where the gap appears to be the largest. The ubiquitous incompressible gaps in the spectra makes the analogy with those in the corresponding two-dimensional systems quite obvious. The novel result here again is, of course, the signature of incompressibility in the energy spectrum for the lowest 


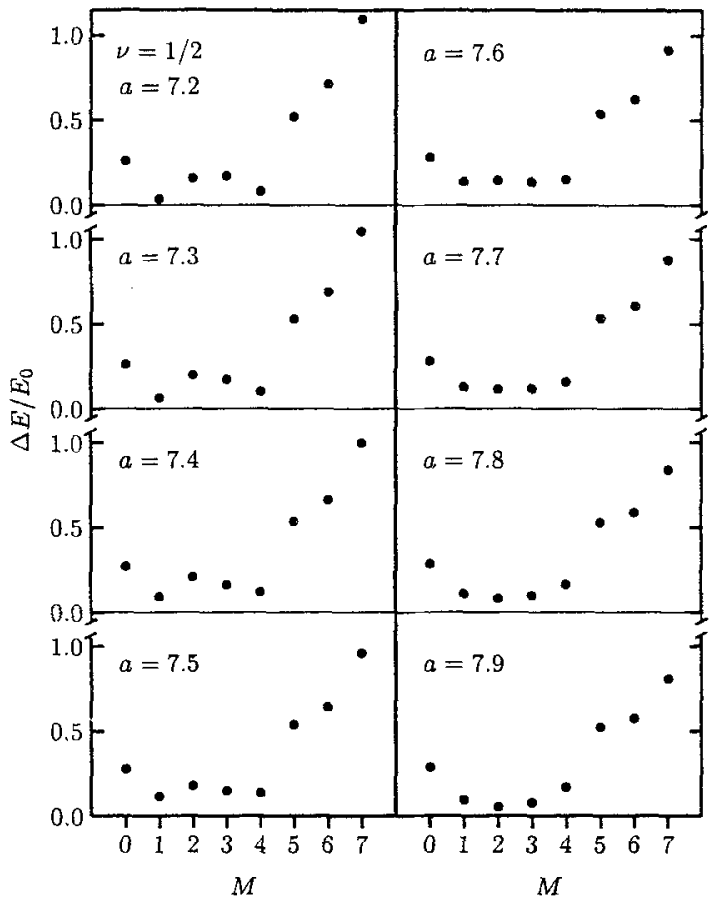

Figure 5

The lowest excitation energy at $v=\frac{1}{2}$ as a function of the total momentum $M$ for various values of $a$.

half-filled Landau level, which is a hallmark of the fractional quantum Hall state at that filling factor [15]. The size of the gap at $v=\frac{1}{2}$ is comparable to that at $\frac{2}{5}$ and should be observable in experiments. There are also experimental indications [11] that the gap size at $v=\frac{1}{2}$ generally depends on the width of the constriction.

In order to explore the areas of the phase diagram where we do not have an incompressible state (between any two stable QH strips in Fig. 3), we looked at the energy spectra of various QH states when $a$ is varied. In Fig. 5, we present such results at $v=\frac{1}{2}$. Evidently, the gap structure changes dramatically from one value of $a$ to another and shows indications of soft modes at certain values of $a$. It therefore indicates that the symmetry changes between the ground states in different regions of the phase diagram. The precise nature of the states with $M \neq 0$ is, as yet, unclear. Similar indications of soft modes were also observed at other filling factors shown in the phase diagram.

\section{Electron Spin Polarizations}

So far, we have not included the role of spin into the problem. In this section we discuss the results for the electron spin polarization in a narrow quantum Hall system [16]. Here we want to describe all possible polarizations $S_{z}$ from 0 to $N_{\mathrm{e}} / 2$. In addition, the noninteracting ground state, as discussed above, must have $M=0$. It should also be noted that the filling factor $v=1$, i.e., one electron per occupied single-particle momentum state should be realized as the ground state for polarized electrons. Similarly, the filling factor $v=2$, i.e., two electrons per occupied state should be realized as the ground state for unpolarized 


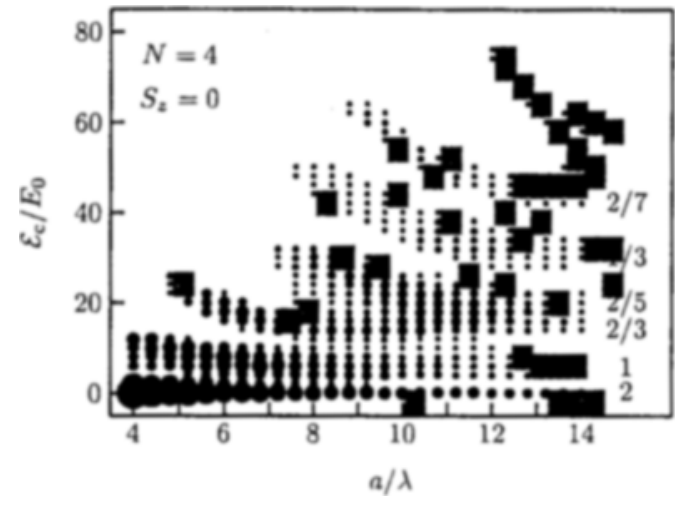

\section{Figure 6}

Phase diagram for electrons in a impurity-free narrow channel quantum Hall system with spin degree of freedom included.

electrons. As a result, the number of electrons $N_{\mathrm{e}}$ must be even. Since we have to apply antiperiodic boundary condition, we should set $N_{\mathrm{e}}=4 n$ where $n$ is an integer. This means that we have to deal with a four electron system because the next natural choice $N_{\mathrm{e}}=8$ is beyond our computational resources. If we exclude the Zeeman energy, there are no spin dependent interactions in the problem and we can set $S_{z}=0$ and get simultaneously the spectrum for $S=0,1, \cdots, N_{e} / 2$.

In Fig. 6, we present the results for the phase diagram, calculated for a system of four electrons with $S_{z}=0$ (Zeeman energy not included) and for $\omega_{0} / \omega_{c}=0.23$, which is appropriate for $B=10$ Tesla and $\hbar \omega_{0}=4 \mathrm{meV}$. As is evident in the figure, several quantum Hall states are stable with large energy gaps in the parameter range considered in this work. In this rather small system the $v=\frac{1}{2}$ state, though supposed to exist, cannot be resolved in the phase diagram. The $v=\frac{1}{2}$ states are expected to lie between $v=\frac{2}{3}$ and $v=\frac{2}{5}$ in Fig. 6. In general, the energy gaps are larger for spinless electrons (Fig. 3) because in that case there are no low-lying spin excitations available.

The temperature dependence of spin polarization for various filling factors found in the phase diagram is calculated by a method we developed earlier [18]. The spin polarization $\left\langle S_{z}(T)\right\rangle$ is calculated from

$$
\left\langle S_{z}(T)\right\rangle \equiv \frac{1}{Z} \sum \mathrm{e}^{-\varepsilon_{j} / k T}\left\langle j\left|S_{z}\right| j\right\rangle
$$

where $Z=\sum_{j} \mathrm{e}^{-\beta \varepsilon_{j}}$ is the canonical partition function and the summation is over all states including all possible polarizations. Here $\varepsilon_{j}$ is the energy of the state $|j\rangle$ with Zeeman coupling included. A direct measurement of $\left\langle S_{z}(T)\right\rangle$ is possible through the NMR Knightshift measurements and also via optical spectroscopic measurements $[17,19]$. These experiments provide unique probes of spin polarizations in the system.

Our results for $\left\langle S_{z}(T)\right\rangle$ vs $T$ at $v=1,2, \frac{2}{3}, \frac{2}{5}, \frac{1}{3}$ and $\frac{1}{2}$ are shown in Fig. 7. In order to obtain the $v=\frac{1}{2}$ results we have employed a six-electron system. In these calculations the magnetic field was kept fixed at 10 tesla and the $g$-factor is varied $(0.02-0.52)$. At $v=1$, we find the results to be similar to those for the 2DES [18] and the system is fully spin polarized even for very low values of the Zeeman energy. Qualitatively similar behavior is also seen at $v=\frac{1}{3}$. In the same way, $v=2$ is a spin-unpolarized state even at the highest value of the Zeeman energy considered and $v=\frac{2}{3}$ and $v=\frac{2}{5}$ are spin-unpolarized states at low Zeeman energies with a non-monotonic temperature dependence as predicted for a 


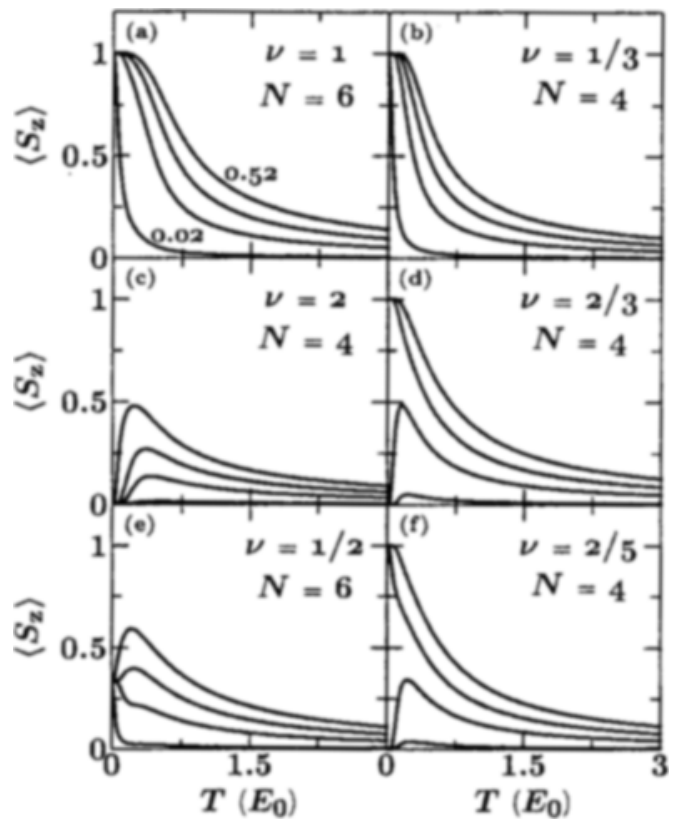

Figure 7

Spin polarization $\left\langle S_{z}\right\rangle$ vs $T$ for $v=1,2, \frac{2}{3}, \frac{1}{2}, \frac{1}{3}$ and $\frac{2}{5}$ for an impurity-free system.

2DEG [18]. Such a non-monotonical behavior is observed in experiments on a 2DES [19]. Clearly, the correspondence with the results of spin polarization in a two dimensional system gives us confidence that our classification of the $\mathrm{QH}$ states in a narrow channel system is essentially correct. At $v=\frac{1}{2}$ we find a spin partially-polarized state.

\section{Laughlin-Like State in a Narrow Channel}

As we mentioned earlier, Yoshioka [12] considered a truncated Coulomb potential, i.e., $v(x, y)=0$ for $|x|>a / 2$, instead of the long-range Coulomb potential. He calculated the overlap between the $\frac{1}{3}$ ground state (spin polarized) and that for a model pseudopotential for which Laughlin state is the ground state [13]. The $\frac{2}{3}-\mathrm{FQH}$ state was then obtained via the electron-hole symmetry: In the $\frac{1}{3} \mathrm{FQH}$ state, $N_{\mathrm{e}}$ electrons occupy the lowest $3 N_{\mathrm{e}}-2$ states. This is the $\frac{2}{3} \mathrm{FQH}$ state where $2\left(N_{\mathrm{e}}-1\right)$ holes occupy the lowest $\left.3 N_{\mathrm{e}}-2\right)$ states. The results [Fig. (8)] indicate that for a suitable choice of parameters, the Laughlin-like $\mathrm{FQHE}$ state for a flat external potential is a good approximation for the ground state even in the presence of the confinement potential. The Laughlin state in a narrow channel is different from the Luttinger liquid state [20] because in the former case a discontinuity exists at the Fermi points. One indeed finds a jump in the momentum distribution function from the occupied state to the unoccupied state. This is because the Laughlin state in the present system has a sharp boundary for the occupation of the single-electron state. In the $1 / m \mathrm{FQHE}$ state with $N_{\mathrm{e}}$ electrons, only the lowest $\left(N_{\mathrm{e}}-1\right) m+1$ states are occupied. However, in an infinitely long system the momentum distribution may not have a discontinuity, and the system may behave like a Luttinger liquid. 


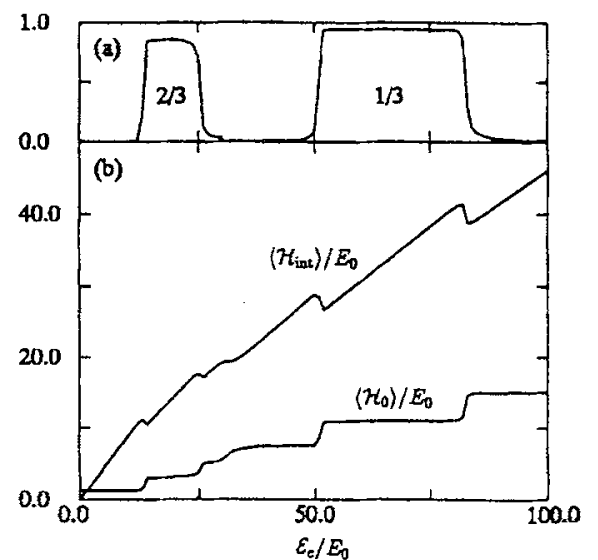

\section{Figure 8}

(a) Overlap of the ground state with the $\frac{1}{3}$ and $\frac{2}{3}$ FQH states, (b) expectation value of the kinetic energy per particle and interaction energy per particle for a truncated Coulomb potential as a function of $\mathcal{E}_{\mathrm{c}} / E_{0}$ for the state $M=0$ and for $a=9.4$ [12].

The Laughlin state in a narrow channel has been also analyzed by the variational Monte Carlo method [21]. For a choice of the periodic potential

$$
v(r)=\lambda\left[\left(\frac{a}{\pi} \sin \frac{\pi}{a} x\right)^{2}+y^{2}\right]^{-\frac{1}{2}},
$$

which reduces to the usual Coulomb potential $\lambda /|r|$ in the limit $x / a \rightarrow 0$, a Laughlin-like wave function was constructed to be

$$
\Psi_{m}\left(z_{1}, \cdots, z_{N_{e}}\right)=\prod_{j<k}\left[\sin \frac{\pi}{a}\left(z_{j}-z_{k}\right)\right]^{m} \prod_{j} \exp \left(-\frac{y_{j}^{2}}{2 \mu \lambda^{2}}\right),
$$

where $z=x+i y / \mu$ is the electron position, $\mu=\ell_{0} / \lambda=\sqrt{1+\left(\omega_{0} / \omega_{c}\right)^{2}}, \ell_{0}=\sqrt{\hbar c / e B}$ and $m$ is an odd integer. In the case of $\mu=1$, i.e., $\hbar \omega_{0} \ll e^{2} /\left(\varepsilon \ell_{0}\right) \ll \hbar \omega_{c}$, a phase diagram at $v=1 / m$ was obtained for a 45 -electron system. Further, a finite-size scaling of the low-lying excitation energies of the system indicated that the correlation function at long distances can be characterized by a non-universal exponent which is in sharp contrast to the standard Laughlin state.

\section{Concluding Remarks}

In this article, we have discussed the electronic properties of incompressible states that are expected to be present in a narrow channel electron system. In between the incompressible states there are states in the phase diagram where the excitation spectra go soft. This indicates a phase transition in going from one $\mathrm{QH}$ state to another. The precise nature of the states in the compressible region is, as yet, unclear. The temperature dependence of electron spin polarization for a narrow quantum Hall system shows behavior analogous to that of a 2DES at major filling factors. At the lowest half-filled quantum Hall state for which no two-dimensional analog exists, a stable spin partially-polarized ground state is expected. Optically pumped NMR experiments as well as optical spectroscopy should be the most appropriate experiments to explore the electronic properties described here. 


\section{Acknowledgments}

I would like to thank Karri Niemelä and Pekka Pietiläinen for their continuing collaboration on these and other related projects. I also thank Peter Fulde for his kind hospitality.

\section{Bibliography}

[1] D.C. Tsui, H.L. Störmer, and A.C. Gossard, Phys. Rev. Lett. 48, 1559 (1982).

[2] R.B. Laughlin, Phys. Rev. Lett. 50, 1395 (1983).

[3] B.I. Halperin, Helv. Phys. Acta 56, 75 (1983).

[4] T. Chakraborty and P. Pietiläinen, The Quantum Hall Effects (Springer, New York, 1995), second edition; T. Chakraborty, in Handbook on Semiconductors, vol. 1, ed. P.T. Landsberg (North-Holland, Amsterdam, 1992), Ch. 17.

[5] H.W. Jiang et al., Phys. Rev. B 40, 12013 (1989); R.L. Willett, et al., Phys. Rev. Lett. 65, 112 (1990); W. Kang et al., ibid. 71, 3850 (1993); R.R. Du et al., ibid., 70, 2944 (1993); ibid., 73, 3274 (1994); D.R. Leadley, et al., ibid., 72, 1906 (1994); R.L. Willett et al., ibid., 75, 2988 (1995).

[6] B.I. Halperin, P.A. Lee, and N. Read, Phys. Rev. B 47, 7312 (1993); B.I. Halperin, Surf. Sci. 305, 1 (1994); B.I. Halperin, in Perspectives in Quantum Hall Effects, edited by S. Das Sarma and A. Pinczuk (Wiley, 1997), ch. 6.

[7] F.D.M. Haldane, Phys. Rev. Lett. 55, 2095 (1985); G. Fano, F. Ortolani, and E. Tosatti, Nuovo Cimento D 9, 1337 (1987); T. Chakraborty and P. Pietiläinen, Phys. Rev. B 38, 10097 (1988); T. Chakraborty, Phys. Rev. B 57, 8812 (1998).

[8] A. Okiji and N. Kawakami, (Eds.), Correlation Effects in Low-Dimensional Electron Systems (Springer, 1994).

[9] S. Tarucha, T. Honda, and T. Saku, Solid State Commun. 94, 413 (1995); A. Yacoby, H.L. Störmer, N.S. Wingreen, L.N. Pfeifier, K.W. Baldwin, and K.W. West, Phys. Rev. Lett. 75, 461 (1996).

[10] S.T. Chui, Phys. Rev. Lett. 56, 2395 (1986).

[11] G. Timp, R. Behringer, J.E. Cunningham, and R.E. Howard, Phys. Rev. Lett. 63, 2268 (1989); G. Timp, in Nanostructured Systems, ed. M. Reed (Academic, Boston, 1992), Ch. 3.

[12] D. Yoshioka, J. Phys. Soc. Jpn. 62, 839 (1993).

[13] D. Yoshioka, Physica B 184, 86 (1993).

[14] T. Chakraborty, Comments Cond. Mat. Phys. 16, 35 (1992); P.A. Maksym and T. Chakraborty, Phys. Rev. Lett. 65, 108 (1990).

[15] T. Chakraborty, K. Niemelä, and P. Pietiläinen, Phys. Rev. Lett. 78, 4829 (1997); T. Chakraborty, K. Niemelä, and P. Pietiläinen, Physica E 1, 80 (1997).

[16] T. Chakraborty, K. Niemelä, and P. Pietiläinen, cond-mat/9711158, and Phys. Rev. B (October $15,1998)$.

[17] S.E. Barrett, et al., Phys. Rev. Lett. 74, 5112 (1995); E.H. Aifer, et al., ibid., 76, 680 (1996).

[18] T. Chakraborty and P. Pietiläinen, Phys. Rev. Lett. 76, 4018 (1996); T. Chakraborty, P. Pietiläinen and R. Shankar, Europhys. Lett. 38, 141 (1997).

[19] I.V. Kukushkin, K. von Klitzing, and K. Eberl, Phys. Rev. B 55, 10607 (1997).

[20] J. Sólyom, Adv. Phys. 28, 201 (1979); J.M. Luttinger, Phys. Rev. 119, 1153 (1960).

[21] S. Tokizaki and Y. Kuramoto, J. Phys. Soc. Jpn. 64, 2302 (1995). 